\title{
Einladung zur Mitgliederversammlung der DRG am Samstag, den 14. November 2020
}

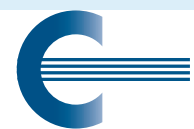

DEUTSCHE RÖNTGENGESELLSCHAFT

Gesellschaft für medizinische Radiologie e.V.

Sehr geehrte Mitglieder der DRG,

im Auftrag des Vorstandes lade ich Sie zur ordentlichen Mitgliederversammlung der Deutschen Röntgengesellschaft - Gesellschaft für Medizinische Radiologie e. V. (DRG), am

Samstag, den 14. November 2020, von 17:00-18:30 Uhr

ein. Nach § 5 (2) des Gesetzes zur Abmilderung der Folgen der COVID-19-Pandemie im Zivil-, Insolvenz- und Strafverfahrensrecht findet die Mitgliederversammlung als Videokonferenz statt.

Es ist folgende Tagesordnung vorgesehen TOP 1: Begrüßung

TOP 2: Totengedenken

TOP 3: Genehmigung des Protokolls der Mitgliederversammlung vom 31. Mai 2019 (veröffentlicht im Jahresbericht 68, Januar 2020)

TOP 4: Bericht des Präsidenten TOP 5: Bericht des Schatzmeisters TOP 6: Bericht der Kassenprüfer TOP 7: Entlastung des Vorstands TOP 8: Wahl der Kassenprüfer 2021
TOP 9: Ergebnisse der zweiten bundesweiten Weiterbildungsumfrage des Forums Junge Radiologie

TOP 10: Einladung zum Deutschen Röntgenkongress 2021

TOP 11: Verschiedenes

Um teilzunehmen, verwenden Sie bitte ein internetfähiges Gerät (PC, Mac, Laptop, Tablet) mit angeschlossenem Mikrofon, Lautsprecher und idealerweise auch Kamera.

Am Veranstaltungstag wählen Sie sich über folgenden Link in die Mitgliederversammlung ein:

https://us02web.zoom.us/j/82035833964? pwd=bzFQNWRUcDhjQ010UVRGMHNhK1 NoZz09

Meeting-ID: 82035833964

Kenncode: 812562

Bitte geben Sie bei der Einwahl unbedingt Ihren richtigen Namen an, damit wir Ihre DRG-Zugehörigkeit prüfen können.

Sofern Sie bisher noch nicht Zoom verwendet haben, installiert sich die Software nachdem Sie auf den Link geklickt haben. Falls Sie bereits Zoom installiert haben, können Sie sich auch über die folgende Meeting-ID einwählen: 8203583 3964. Eine Einwahl über eine Festnetztelefonnummer bieten wir nicht an, da damit keine Teilnahme an elektronischen Abstimmungen möglich ist.

Da es sich um eine Videokonferenz handelt, werden Sie auch für die anderen Teilnehmer*innen zu sehen sein, sofern Ihr Gerät über eine Kamera verfügt. Sie können die Kamera in der Software ausschalten, falls Sie nicht gesehen werden möchten.

Falls Sie Fragen zur Technik haben, können Sie sich gern telefonisch oder per Email an die DRG-Geschäftsstelle wenden (Tel.: 030916 070-0, Mail: office@drg.de)

Zum Schutz Ihrer personenbezogenen Daten hat die DRG einen Auftragsdatenverarbeitungsvertrag mit Zoom abgeschlossen. Zoom verpflichtet sich darin, Ihre Daten nur innerhalb Europas DSGVO-Konform zu verarbeiten. Zoom ist damit Dienstleister der DRG.

Mit freundlichen Grüßen

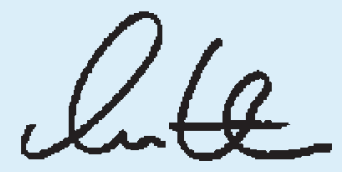

Prof. Dr. Gerald Antoch Präsident 